\title{
USO DE TRIAMCINOLONA Y BEVACIZUMAB EN \\ CIRUGÍA DE FACO-VITRECTOMÍA 25G PARA EL TRATAMIENTO DE CATARATA Y EDEMA MACULAR DIABÉTICO
}

\section{USE OF TRIAMCINOLONE AND BEVACIZUMAB IN 25G PHACO-VITRECTOMY SURGERY FOR THE TREATMENT OF CATARACT AND DIABETIC MACULAR EDEMA}

\section{RESUMEN}

Objetivo: Evaluar la seguridad y eficacia de la cirugía combinada de facoemulsificación y vitrectomía asistida con triamcinolona y aplicación de bevacizumab intravítreo en pacientes con catarata y edema macular diabético difuso.

Pacientes y métodos: Estudio prospectivo, longitudinal, no aleatorizado, ensayo clínico no controlado. Se incluyeron trece pacientes en forma consecutiva que presentaron esencialmente catarata subcapsular posterior y edema macular difuso, a los que se les realizó cirugía de facoemulsificación, vitrectomía a tres puertos $25 \mathrm{G}$ asistida con triamcinolona, endofotocoagulación y aplicación de bevacizumab intravítreo. Se realizó seguimiento con medición de agudeza visual, fluorangiografía retiniana y tomografía óptica coherente (OCT) basal, a las 2 semanas, en el primer, tercero y sexto meses.

Resultados: Se encontró una mejoría visual de al menos 2 líneas de visión al final del seguimiento en diez de los pacientes. En cuanto al grosor central
Objective: To evaluate the safety and efficacy of combined phacoemulsification and triamcinoloneassisted vitrectomy with intravitreal injection of bevacizumab in patients with cataract and diffuse diabetic macular edema.

Methods: In this prospective, non randomized, interventional case series we included 13 eyes of 13 patients with posterior subcapsular cataracts and diffuse macular edema who underwent phacoemulsification, 25G triamcinolone-assisted vitrectomy, endophotocoagulation and had a bevacizumab injection at the end of the procedure. The follow-up consisted of basal best corrected visual acuity, fluorescein angiography, and optical coherent tomography performed at 2 weeks, one, three and six months post-operatively.

Results: An improvement in the best corrected visual acuity of at least two lines was seen in 10 of the 13 patients. The basal average central macular thickness initially was $358.23 \mu$ (SD $50 \mu$ ) while at the end of follow-up was $216.6 \mu$ (SD $23.2 \mu$ ).

\footnotetext{
Recibido: 10/8/07. Aceptado: 11/3/08.

Unidad de Oftalmología. Clínica David. Morelia, Mich, México.

1 Doctor en Medicina.

Correspondencia:

Sergio Eustolio Hernández Da Mota

Unidad de Oftalmología. Clínica David

Blvd. García de León, 598

Colonia Nueva Chapultepec

Morelia, Michoacán

58280 México

E-mail: tolodamota@yahoo.com.mx
} 
macular, el promedio fue de 358,23 $\mu$ (DS 56,0 $\mu$ ) preoperatoriamente, mientras que al final del seguimiento fue de 216,6 $\mu$ (DS 23,2 $\mu$ ).

Conclusiones: El tratamiento quirúrgico y farmacológico combinado aquí expuesto en el periodo de seguimiento de seis meses, mostró una reducción significativa en el grosor macular así como mejoría de agudeza visual en la mayoría de los pacientes. Estudios comparativos con series mas grandes serán necesarios para establecer la eficacia real del procedimiento a largo plazo.

Palabras clave: Catarata, facoemulsificación, vitrectomía, triamcinolona, bevacizumab.
Conclusion: This combined surgical and pharmacological treatment resulted in an improvement of central macular thickness and non-progression of diabetic retinopathy in the six month follow-up period. Larger comparative studies are necessary to establish the real impact of this therapeutic approach (Arch Soc Esp Oftalmol 2008; 83: 293-300).

Key words: Cataract, phacoemulsification, vitrectomy, triamcinolone, bevacizumab.

\section{INTRODUCCIÓN}

La retinopatía diabética es una complicación tardía de la diabetes mellitus, así como la formación de catarata a la que se le ha denominado metabólica. La duración de la enfermedad, el inadecuado control de la glicemia, entre otros, son factores que aceleran la aparición de estas condiciones (1-3).

La cirugía de catarata merece una especial mención ya que los pacientes con diabetes mellitus sometidos a este procedimiento, muestran una progresión de la retinopatía diabética y muchas veces también una aparición de edema macular o incremento del mismo en caso de estar ya presente (4-6). La conducta establecida en muchos de estos casos es la aplicación de fotocoagulación en la mácula previa a la cirugía cuando la opacidad del cristalino permita realizarla o inmediatamente después (7-9). Sin embargo, en muchos casos no se consigue controlar o disminuir el engrosamiento macular y los resultados visuales en el largo plazo son más bien pobres (10). Otros tratamientos empleados en años recientes, han sido: esteroides intravítreos como la triamcinolona) (11-23), fármacos antiangiogénicos (bevacizumab) $(24,25)$ y recursos quirúrgicos (vitrectomía) (26-28). Todos los anteriores empleados en forma aislada tienen sus ventajas; pero al mismo tiempo, tienen ciertos riesgos así como también efectos transitorios como en el caso de los fármacos intravítreos (29-32).

El propósito de este estudio es describir una técnica que combine en un mismo tiempo, las opciones de tratamiento quirúrgico mínimamente invasivo (vitrectomía 25G) y farmacológico, sin el efecto de la energía térmica láser en el área macular, así como evaluar su eficacia, seguridad y resultados en una serie piloto de trece pacientes durante seis meses.

\section{SUJETOS, MATERIAL Y METODO}

Se pidió consentimiento informado a cada uno de los pacientes incluídos en el estudio. Los criterios de inclusión fueron: pacientes con retinopatía diabética no proliferativa y edema macular difuso según los criterios establecidos por el ETDRS (Early Treatment Diabetic Retinopathy Study), catarata subcapsular posterior grado II según la clasificación LOCS (Lens Opacities Classification System) P1 a P2, control metabólico adecuado por al menos 3 meses previos al procedimiento, considerado como el tener hemoglobina glicosilada $(\mathrm{Hb} 1 \mathrm{aC})$ con cifras menores a 7. También se requirió control de tensión arterial definido como una tensión arterial por debajo de 140/90 mmHg durante al menos 3 meses previos a la intervención quirúrgica. Los criterios de exclusión fueron: pacientes con control metabólico inadecuado, insuficiencia renal, hipertensión arterial descontrolada, retinopatía diabética proliferativa, opacidad del cristalino que no fuera la clasificada según la establecida por la clasificación LOCS previamente mencionada. A todos los pacientes se les realizó toma de agudeza visual basal corregida con cartilla de escala decimal, biomicroscopía de segmento anterior y de segmento posterior con lente de tres espejos de Goldman, toma de presión intraocular (PIO), examen de fondo de ojo, fluorangiografía y tomografía óptica coherente utilizando la estrategia «fast macular» (mapeo macular) basales, al segundo día de posto- 
peratorio, a las 2 semanas, 1 mes, 3 y 6 meses de realizado el procedimiento completando así el seguimiento.

La técnica quirúrgica consistió en primer lugar, en realizar facoemulsificación en un primer tiempo con técnica de «stop and chop» (parar y cortar) (fig. 1), colocación de lente intraocular monobloque acrílica Acrysoft (Alcon laboratorios, Inc. Huntington West, Virginia, EUA), dejando el material viscoelástico en cámara anterior para posteriormente proceder a la realización de vitrectomía a tres puertos $25 \mathrm{Ga}$, central, con el equipo Accurus (ALCON, Fort Worth, Tx, EUA), endoiluminación con luz de xenón, sistema de visualización panorámica BIOM (Insight instrumets, Sanford, Fl) (fig. 2).

Se procedió a realizar vitrectomía a 1.500 cortes por minuto y $200 \mathrm{mmHg}$ de aspiración previa aplicación de triamcinolona libre de conservadores 1 $\mathrm{mg}$ en $0,1 \mathrm{ml}$ (ATLC, Laboratorios Grin, México, DF, México) para impregnar el vítreo central y hialoides posterior (fig. 3), se realizó primero vitrectomía central y luego separación de la hialoides posterior lo cual se efectuó mediante aspiración simple con la cabeza del vitrector aumentando la aspiración hasta $350 \mathrm{mmHg}$. Posteriormente se procedió a realizar endofotocoagulación dispersa con láser de diodo fuera del área macular con un promedio de 800 impactos de aproximadamente $500 \mu$ de diámetro (IRIS medical, Stanford, California, E.U.A). Se retiraron los puertos de entrada y se procedió a la inyección intravítrea de 0,05 ml (1,25 mg) de Bevacizumab (Avastin, Laboratorios Roche, México, D.F., México) (fig. 4), en el cuadrante temporal

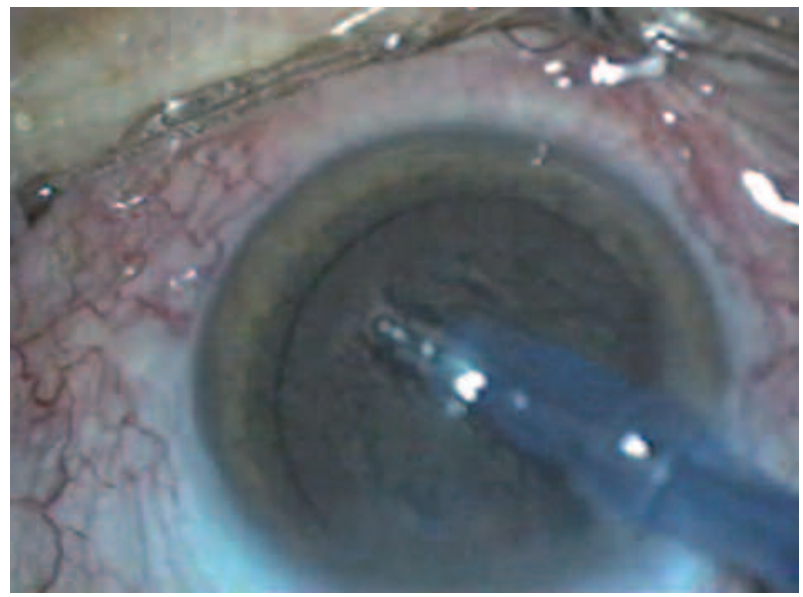

Fig. 1: Facoemulsificación con técnica de «stop and chop» (parar y cortar).

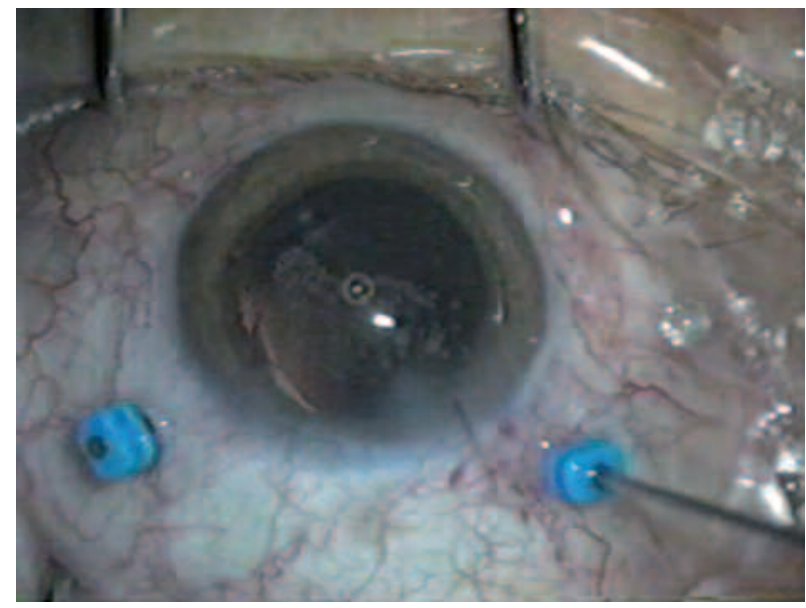

Fig. 2: Vitrectomía calibre 25.

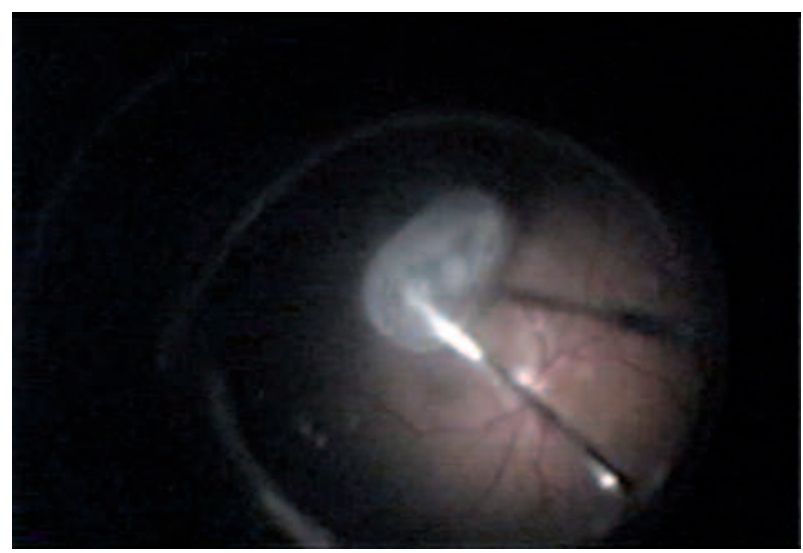

Fig. 3: Inyección intravítrea de triamcinolona.

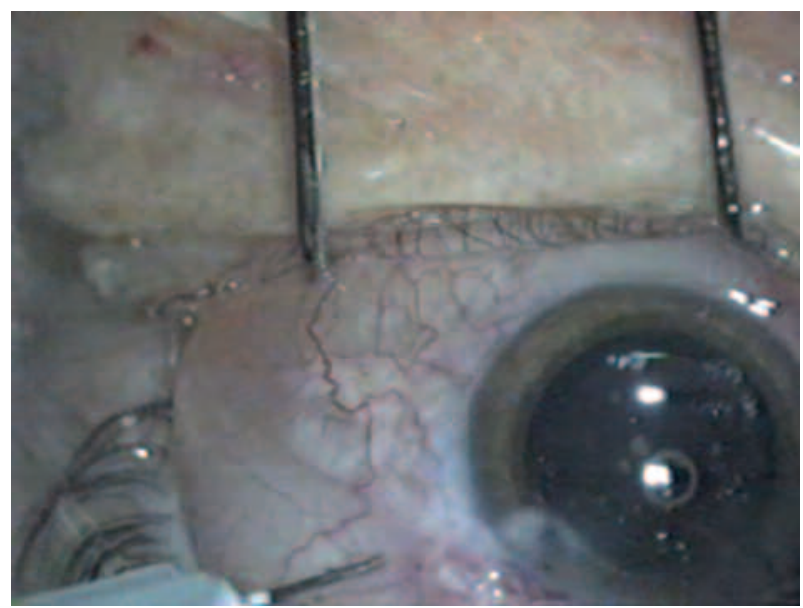

Fig. 4: Inyección de bevacizumab (AVASTIN) intravítreo. 
superior a $3 \mathrm{~mm}$ del limbo esclerocorneal. Posteriormente se extrajo el material viscoelástico mediante aspiración con la sonda de irrigación y aspiración. En el seguimiento postoperatorio se empleó una combinación de tobramicina y dexametasona en suspensión para aplicación tópica durante 3 semanas 4 veces al día.

\section{RESULTADOS}

Trece ojos de trece pacientes fueron incluídos en este estudio. Las edades de los pacientes estaban comprendidas en un rango de entre 40 a 78 años con un promedio de 58 años. Cinco pacientes eran del sexo masculino. Las agudezas visuales preoperatorios variaron de MM (movimiento de manos) a 20/70. Las presiones intraoculares preoperatorias tuvieron a su vez, un rango de 12 a $20 \mathrm{mmHg}$. Se observó aumento de la PIO mayor de $20 \mathrm{mmHg}$ en seis pacientes al primer día de postoperatorio. En todos ellos se controló con una combinación de maleato de timolol al 0,5\% y dorzolamida. En la segunda semana de seguimiento, tres pacientes presentaron una PIO mayor $20 \mathrm{mmHg}$. A partir del mes, ninguno de los pacientes mostró cifras tensionales altas suspendiendo la medicación antiglaucomatosa.

El gráfico 1 muestra las agudezas visuales basales y a los 6 meses de haberse realizado el procedimiento.

El promedio de grosor central foveal fue $358,23 \mu$ con un rango de 285 a $503 \mu$ en el segundo día de postoperatorio. Al final del seguimiento (6 meses) el promedio de grosor foveal fue de 215,6 $\mu$ con un rango de 198 a $280 \mu$ (gráf. 2).

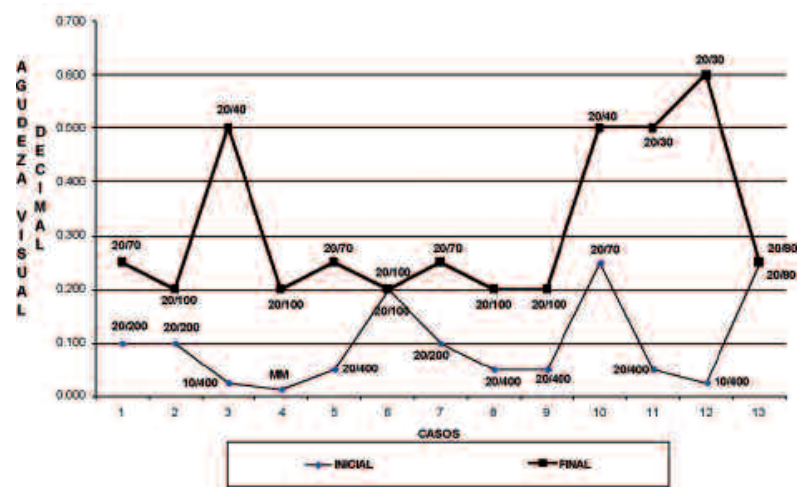

Gráf. 1: Comparación inicial y final de la agudeza visual.

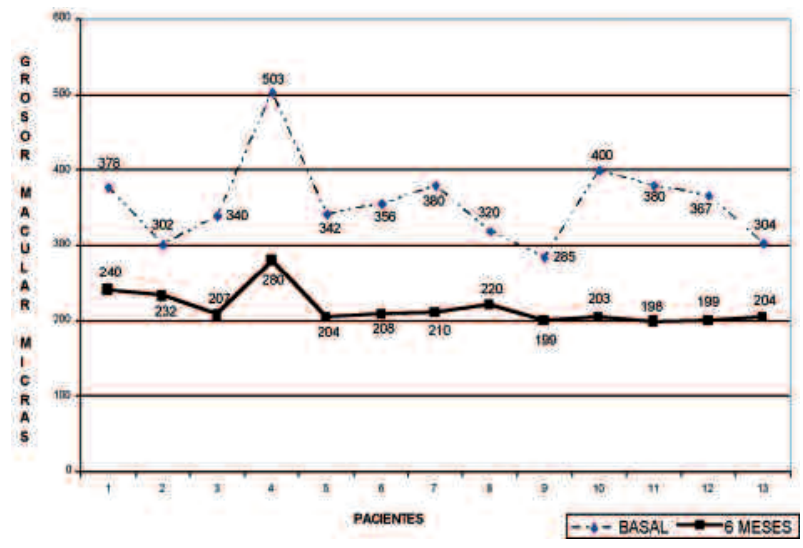

Gráf. 2: Comparación del OCT basal y 6 meses.

Durante el seguimiento, no se observó desprendimiento de retina, hemorragia vítrea, neovascularización de iris y/o ángulo en ninguno de los pacientes.

\section{DISCUSIÓN}

Los resultados obtenidos en nuestra publicación, muestran una disminución significativa del grosor central macular, documentada por OCT, la cual se mantuvo a lo largo de todo el seguimiento de los pacientes. En cuanto a la agudeza visual, se logró mejoría de la misma en 11 de los 13 pacientes estudiados. Y en diez de ellos se logró documentar una mejoría de por lo menos 2 líneas al final del seguimiento. Comparado con publicaciones como el de Oshima y colaboradores (28), la mejoría fue menor en el nuestro, aunque en aquel se incluyeron pacientes sin patología macular. Por otro lado, en el artículo de La Heij (27) se obtuvo un rango de mejoría visual de 0,25 a 0,5 mientras que en nuestro estudio fue de 0,25 a 0,6 , siendo, aunque marginal, mejor la obtenida por nosotros.

El propósito de nuestro artículo fue realizar un procedimiento en pacientes que cursaban con catarata y edema macular diabético combinando las alternativas que prescindieran del láser aplicado en el área macular, con el objetivo de determinar la evolución de esta serie de pacientes en un periodo de 6 meses. A pesar de que en artículos como el de Patel y colaboradores (26), no se apreció ninguna diferencia entre la terapia con láser y la vitrectomía vía pars plana en el tratamiento del edema macular, pensamos que agregar triamcinolona y bevacizumab junto con la vitrectomía pudiera dar mejores 
resultados funcionales que se mantuvieran en el largo plazo.

El uso de triamcinolona ha sido tema de múltiples publicaciones (11-23) en donde se ha visto un beneficio tangible en la reducción del grosor macular y en algunos casos mejoría en la agudeza visual, sobretodo en los estudios iniciales (11-14). En ellos, se empleó para el tratamiento de edema macular refractario a laserterapia; sin embargo, el efecto del medicamento sigue siendo temporal (20) y en muchas ocasiones se requieren reinyecciones con los riesgos que esto implica (hipertensión ocular, desprendimiento de retina, hemorragia vítrea, endoftalmitis tanto estéril como infecciosa) (29-32). En otros casos, se ha empleado como adyuvante en la cirugía vitreoretiniana para visualizar mejor la hialoides posterior y poder extraerla con mas eficacia $(33,34)$. En nuestro estudio decidimos usar una dosis menor a $2 \mathrm{mg}$ con la hipótesis de que disminuiríamos la incidencia de hipertensión ocular pero conservando su efecto para impregnar la hialoides posterior y así poder extraerla con mayor facilidad así como disminuir el grosor macular; sin embargo, la hipertensión ocular se presentó, aunque de forma transitoria (1 mes), en seis de nuestros pacientes.

Otro de los tratamientos alternativos a la fotocoagulación ya mencionados en los pacientes que cursan concomitantemente con catarata y edema macular diabético es la realización de vitrectomía. Con el advenimiento de la tomografía óptica coherente, en muchos casos de edema macular se ha podido documentar el llamado síndrome de tracción vitreoretiniana que se ha visto implicado en dicho edema. Al realizar la vitrectomía, sobre todo con extracción de la hialoides posterior (que en algunos casos por no visualizarla no se logra) el edema cede y puede haber mejoría del engrosamiento macular y de la agudeza visual $(23,34)$. Hay artículos incluso, en donde aún no habiendo evidencia de síndrome de tracción vitreomacular, el hecho de realizar vitrectomía puede producir una mejoría en el edema y la función macular (27), probablemente por la remoción de factores proangiogénicos como el factor de crecimiento vascular endotelial (VEGF por sus siglas en inglés) y de citoquinas involucradas en la ruptura de la barrera hematorretiniana, así como el aumento de los niveles de oxígeno en la cavidad vítrea (35). Dicho factor se haya implicado en la ruptura de la barrera hematoretiniana y en el subsecuente mantenimiento y/o empeoramiento del edema en el área macular.
La vitrectomía como tal, no se encuentra exenta de complicaciones; desprendimiento de retina, sobre todo cuando se realiza con instrumentos del calibre 20 (se ha comunicado una menor incidencia de desprendimiento de retina con instrumentos de calibre 25) (28), neovascularización de iris y ángulo, con el consabido glaucoma neovascular que termina muchas veces con la visión del paciente, son algunas de ellas, aún y cuando la introducción de los sistemas de endofotocoagulación han bajado mucho las tasas de éste. La rubeosis se puede deber, en parte, a que persisten niveles significativamente altos del VEGF una vez hecho el procedimiento quirúrgico (36). En nuestra publicación no se observaron estas complicaciones durante todo el seguimiento, por el empleo quizás de un calibre pequeño, que no permite tan frecuentemente la herniación del vítreo por los puertos de entrada y por lo tanto se disminuye el riesgo de desprendimiento de retina, así como la menor reacción inflamatoria $(28,33)$. Sin embargo la muestra de nuestro artículo, probablemente no sea representativa para apoyar dicha aseveración por ser un estudio piloto. El otro factor que pensamos disminuye el riesgo de desarrollo de rubeosis es la aplicación concomitante del fármaco antiangiogénico, bevacizumab.

$\mathrm{Al}$ respecto, el bevacizumab, se ha reportado que produce mejoría en el edema macular $(24,25)$. Sin embargo, la aplicación intravítrea de este medicamento también debe ser en forma repetida debido a su efecto también transitorio conllevando riesgos, al igual que la inyección de triamcinolona, tales como: desprendimiento de retina, hemorragia vítrea y endoftalmitis (25). En nuestra serie lo empleamos con un doble propósito: el de disminuir la incidencia de glaucoma neovascular, al bloquear la acción de los niveles de VEGF presentes en la cavidad vítrea después del procedimiento y el de actuar hipotéticamente en forma aditiva tanto en efecto como en duración con la vitrectomía y la triamcinolona en disminuir el grosor central macular.

El empleo de esta terapia combinada quirúrgicafarmacológica mostró ser segura en nuestro grupo de pacientes, eficaz en la reducción del grosor central macular así como en mejorar la agudeza visual en la mayoría de los casos y mantenerla estable en un periodo de seis meses. En conclusión, pretendimos mostrar una alternativa de las modalidades de tratamiento vigentes, que evitara por periodos más prolongados inyecciones intravítreas repetidas (con los efectos adversos conocidos de desprendimiento 
de retina, endoftalmitis, hemorragia vítrea, etc.), quemaduras térmicas maculares por láser y que disminuyera los riesgos inherentes a la vitrectomía, (como el desprendimiento de retina) usando instrumentos de calibre pequeño (25 G). Sin embargo, serán necesarios artículos comparativos, con series más grandes para establecer la viabilidad de esta técnica en el tratamiento de los pacientes en donde coexista catarata y edema macular diabético.

\section{BIBLIOGRAFÍA}

1. The effect of intensive treatment of diabetes on the development and progression of long-term complications in insulin-dependent diabetes mellitus. Diabetes Control and Complications Trial Research Group. N Eng J Med 1993; 329: 977-986.

2. The relationship on glycemic exposure (HbAlc) to the risk of development and progression of retinopathy in the diabetes control and complications trial. Diabetes 1995; 44 : 968-983.

3. The absence of a glycemic threshold for the development of long term complications: the perspective of the Diabetes Control and Complications Trial. Diabetes 1996; 45 : 1289-1298.

4. Flesner P, Sander B, Henning V, Parving HH, Dornonville de la Cour M, Lund-Andersen H. Cataract surgery on diabetic patients. A prospective evaluation of risk factors and complications. Acta Ophthalmol Scand 2002; 80: 19-24.

5. Gupta A, Gupta V. Diabetic maculopathy and cataract surgery. Ophthalmol Clin North Am 2001; 14: 625-637.

6. Squirrell D, Bhola R, Bush J, Winder S, Talbot JF. A prospective, case controlled study of the natural history of diabetic retinopathy and maculopathy after uncomplicated phacoemulsification cataract surgery in patients with type 2 diabetes. Br J Ophthalmol 2002; 86: 565-571.

7. Photocoagulation for diabetic macular edema Early Treatment Diabetic Retinopathy Study report number 1. Early Treatment Diabetic Retinopathy Study Research Group. Arch Ophthalmol 1985; 103: 1796-1806.

8. Photocoagulation for diabetic macular edema: Early Treatment Diabetic Retinopathy Study report no. 4. Early Treatment Diabetic Retinopathy Study Research Group. Int Ophthalmol Clin 1987; 27: 265-272.

9. Treatment techniques and clinical guidelines for photocoagulation of diabetic macular edema. Early Treatment Diabetic Retinopathy Study Report Number 2. Early Treatment Diabetic Retinopathy Study Research Group. Ophthalmology 1987; 94: 761-774.

10. Han DP, Miller WF, Burton TC. Submacular fibrosis after photocoagulation for diabetic macular edema. Am J Ophthalmol 1992; 113: 513-521.

11. Martidis A, Duker JS, Greenberg PB, Rogers AH, Puliafito $C A$, Reichel $E$, et al. Intravitreal triamcinolone for refractory diabetic macular edema. Ophthalmology 2002; 109:920-927.

12. Jonas JB, Kreissig I, Söfker A, Degenring RF. Intravitreal injection of triamcinolone for diffuse diabetic macular edema. Arch Ophthalmol 2003; 121: 57-61.
13. Massin P, Audren F, Haouchine B, Erginay A, Bergmann $J F$, Benosman $R$, et al. Intravitreal triamcinolone acetonide for diabetic diffuse macular edema: preliminary results of a prospective controlled trial. Ophthalmology 2004; 111: 218-225.

14. Sakamoto T, Miyazaki M, Hisatomi T, Nakamura T, Ueno $A$, Itaya $K$, et al. Triamcinolone-assisted pars plana vitrectomy improves the surgical procedures and decreases the postoperative blood-ocular barrier breakdown. Graefes Arch Clin Exp Ophthalmol 2002; 240: 423-429.

15. Pinheiro A, Serracarbassa PD, Maia OO Jr, Takahashi WY. Macular thickness analysis by optic coherence tomography in refractory diabetic macular edema treated with intravitreous triamcinolone. Arq Bras Oftalmol 2007; 70: 784-789.

16. Ciardella AP, Klancnik J, Schiff W, Barile G, Langton K, Chang $S$. Intravitreal triamcinolone for the treatment of refractory diabetic macular oedema with hard exudates: an optical coherence tomography study. Br J Ophthalmol 2004; 88: 1131-1136.

17. Jonas JB, Kreissig I, Degenring RF, Kamppeter BA. Repeated intravitreal injection of triamcinolone acetonide for diffuse diabetic macular oedema. Br J Ophthalmol 2005; 89: 122.

18. Gómez-Ulla F, Marticorena J, Alfaro DV 3rd, Fernández $M$, Méndez ER, Rothen M. Intravitreal triamcinolone for the treatment of diabetic macular edema. Curr Diabetes Rev 2006; 2: 99-112.

19. Degenring RF, Kreissig I, Jonas JB. Intraocular triamcinolone for diffuse diabetic macular edema. Ophthalmologe 2004; 101: 251-254.

20. Jonas JB, Degenring RF, Kamppeter BA, Kreissig I, Akkoyun I. Duration of the effect of intravitreal triamcinolone acetonide as treatment for diffuse diabetic macular edema. Am J Ophthalmol 2004; 138: 158-160.

21. Ozkiris A, Evereklioglu C, Erkiliç K, Tamçelik N, Mirza E. Intravitreal triamcinolone acetonide injection as primary treatment for diabetic macular edema. Eur J Ophthalmol 2004; 14: 543-549.

22. Chan CK, Mohamed S, Shanmugam MP, Tsang CW, Lai TY, Lam DS. Decreasing efficacy of repeated intravitreal triamcinolone injections in diabetic macular oedema. Br J Ophthalmol 2006; 90: 1137-1141.

23. Jonas JB, Söfker A, Degenring R. Intravitreal triamcinolone acetonide as an additional tool in pars plana vitrectomy for proliferative diabetic retinopathy. Eur J Ophthalmol 2003; 13: 468-473.

24. Ferrara N, Hillan KJ, Novotny W. Bevacizumab (Avastin), a humanized anti-VEGF monoclonal antibody for cancer therapy. Biochem Biophys Res Commun 2005; 333: 328335.

25. Arevalo JF, Fromow-Guerra J, Quiroz-Mercado H, Sanchez JG, Wu L, Maia M, et al. Primary intravitreal bevacizumab (Avastin) for diabetic macular edema: results from the Pan-American Collaborative Retina Study Group at 6month follow-up. Ophthalmology 2007; 114: 743-750.

26. Patel JL, Hykin PG, Schadt M, Luong V, Fitzke F, Gregor ZJ. Diabetic macular oedema: pilot randomized trial of pars plana vitrectomy vs macular argon photocoagulation. Eye 2006; 20: 873-881.

27. La Heij EC, Hendrikse F, Kessels AG, Derhaag PJ. Vitrectomy results in diabetic macular oedema without 
evident vitreomacular traction. Graefes Arch Clin Exp Ophthalmol 2001; 239: 264-270.

28. Oshima Y, Ohji M, Tano Y. Surgical outcomes of 25-gauge transconjunctival vitrectomy combined with cataract surgery for vitreoretinal diseases. Ann Acad Med Singapore 2006; 35: 175-180.

29. Jaissle GB, Szurman P, Bartz-Schmidt KU. Ocular side effects and complications of intravitreal triamcinolone acetonide injection. Ophthalmologe 2004; 101:121-128.

30. Lecleire-Collet A, Offret O, Gaucher D, Audren F, Haouchine B, Massin P. Full-thickness macular hole in a patient with diabetic cystoid macular oedema treated by intravitreal triamcinolone injections. Acta Ophthalmol Scand 2007; 85: 795-798.

31. Gillies MC, Kuzniarz M, Craig J, Ball M, Luo W, Simpson $J M$. Intravitreal triamcinolone-induced elevated intraocular pressure is associated with the development of posterior subcapsular cataract. Ophthalmology 2005; 112: 139-143.
32. Moshfeghi DM, Kaiser PK, Scott IU, Sears JE, Benz M, Sinesterra JP, et al. Acute endophthalmitis following intravitreal triamcinolone acetonide injection. Am J Ophthalmol 2003; 136: 791-796.

33. Gupta OP, Weichel ED, Regillo CD, Fineman MS, Kaiser RS, Ho AC, et al. Postoperative complications associated with 25-gauge pars plana vitrectomy. Ophthalmic Surg Lasers Imaging 2007; 38: 270-275.

34. Doi N, Uemura A, Nakao K, Sakamoto T. Vitreomacular adhesion and the defect in posterior vitreous cortex visualized by triamcinolone-assisted vitrectomy. Retina 2005; 25: $742-745$.

35. Steffánson E. The therapeutic effects of retinal laser treatment and vitrectomy. A theory based on oxygen and vascular phisiology. Acta Ophtalmol Scand 2001; 79: 435-440.

36. Itakura $\mathrm{H}$, Kishi S, Kotajima N, Murakami M. Persistent secretion of vascular endothelial growth factor into the vitreous cavity in proliferative diabetic retinopathy after vitrectomy. Ophthalmology 2004; 111: 1880-1884. 